\title{
AVALIAÇÃO DA QUALIDADE DAS ANOTAÇÕES DE ENFERMAGEM EM UNIDADE SEMI-INTENSIVA
}

\author{
Quality assessment of nursing records in a semi-intensive care unit
}

Evaluaión de la calidad de las anotaciones de enfermería en una unidad semeintensiva

Josy Anne Silva ${ }^{1}$

Ana Cândida Martins Grossi

Maria do Carmo Lourenço Haddad ${ }^{3}$

Sonia Silva Marcon ${ }^{4}$

\section{RESUMO}

A anotação de enfermagem é um dos meios para se avaliar os cuidados prestados. 0 objetivo do estudo foi avaliar a qualidade das anotações de enfermagem em uma unidade semi-intesiva. Os dados, coletados com um roteiro para auditoria retrospectiva, são referentes a 16 pacientes que aguardavam vaga para Unidade de Terapia Intensiva, internados em dezembro de 2009 e janeiro de 2010, por um período médio de oito dias. No quesito identificação, o percentual de preenchimento foi de $74,8 \%$ o que é bem próximo dos valores considerados satisfatórios ( $80 \%$ ); porém o percentual de preenchimento completo dos itens: registros, procedimentos e prescrição de enfermagem, terapia intensiva e execução de ordens médicas alcançaram respectivamente $54,7 \%, 41,1 \%, 39,3 \%, 34,9 \%$ e $25 \%$. Os baixos índices de preenchimento completo revelam falha grave no registro da assistência prestada nesta unidade, o que pode estar prejudicando a continuidade do cuidado e a legitimação do trabalho da enfermagem.

Palavras-chave: Registros de enfermagem. Auditoria de enfermagem. Qualidade da assistência à saúde. Cuidados intensivos.

\begin{abstract}
Nursing records can be used as a method to assess quality of care. The aim of this study was to assess quality of nursing records in a semi-intensive care unit. Data were collected using a guide for retrospective audit, gathering information from 16 patients awaiting admittance into an Intensive Care Unit, admitted in the period of December 2009 to January 2010, with an average of 8 days of hospitalization. Regarding identification, the percentage of filled in records was $74.8 \%$, which is close to acceptable standards (80\%). However, the percentage of complete filled in items were respectively: nursing records, procedures and nursing prescription, intensive care and execution of medical orders accounting for $54.7 \%, 41.1 \%$, $39.3 \%, 34.9 \%$ and $25 \%$. The reduced levels of complete filled in records reveal a major flaw in recording care provided in this unit which may be hindering the continuance of care and is prejudicial to the legitimization of care provided by nurses.
\end{abstract}

Keywords: Nursing records. Nursing Audit. Quality of Health Care. Intensive Care.

\section{Resumen}

La anotación de la enfermería es un medio de evaluar la atención recibida. El objetivo de este estudio fue evaluar la calidad de las notas de enfermería en una unidad semeintensiva. Los datos, recogidos con una hoja de ruta para la auditoría retrospectiva, se refieren a 16 pacientes en espera de vacante para la Unidad de Cuidados Intensivos, admitió en diciembre de 2009 y enero de 2010, para un promedio de ocho días. Sobre la identificación, el porcentaje de avance fue de $74,8 \%$, lo cual es muy similar a los valores considerados satisfactorios $(80 \%)$, pero el porcentaje de llenado completo de los artículos, los registros, procedimientos y la prescripción de enfermería, cuidados intensivos y la ejecución de las órdenes del médico llegaron, respectivamente, a $54,7 \%, 41,1 \%, 39,3 \%, 34,9 \%$ y un $25 \%$. Las bajas tasas de llenado completo del registro revelan incumplimiento grave de la atención en esta unidad, que puede ser perjudicial para la continuidad de la atención y la legitimación del trabajo de enfermería.

Palabras clave: Registros de Enfermería. Auditoria de Enfermería. Calidad de la Atención de Salud. Cuidados Intensivos.

\footnotetext{
${ }^{1}$ Enfermeira do Hospital Universitário de Maringá. Especialista em farmacologia. Docente do Curso de Graduação em Enfermagem da Faculdade Ingá. Maringá-PR. Brasil. E-mail: josy.a.s@uol.com.br; ${ }^{2}$ Enfermeira. Mestre em Enfermagem pela Universidade Estadual de Maringá (UEM). Docente do curso de Enfermagem da Universidade Estadual do Norte do Paraná (UENP), Campus Luiz Meneghel. Bandeirantes-PR. Brasil. E-mail: anacandidagrossi@ffalm.br; ${ }^{3}$ Enfermeira. Doutora em Fundamentos da Enfermagem. Docente da graduação e pós-graduação em Enfermagem na Universidade Estadual de Londrina (UEL). Londrina - PR. Brasil. E-mail: Haddad@sercomtel.com.br; ${ }^{4}$ Enfermeira. Doutora em Filosofia da Enfermagem. Docente da graduação e pósgraduação em enfermagem da UEM. Maringá-PR. Brasil. E-mail: soniasilva.marcon@gmail.com
} 


\section{INTRODUÇÃO}

Considerando que $50 \%$ das informações referentes ao cuidado são fornecidas pela enfermagem, espera-se que os registros realizados por esta categoria permitam a comunicação permanente entre os membros da equipe multiprofissional, com transmissão de informações que facilitem o planejamento, tomada de decisões clínicas e gerenciais e continuidade da assistência prestada ${ }^{1}$.

A anotação de enfermagem consiste em um importante meio de comunicação para a equipe, pois além de indicar as ações realizadas, possibilita uma sequência na continuidade da assistência ${ }^{2}$. No âmbito hospitalar, por exemplo, ela compreende o registro da evolução do paciente durante a internação hospitalar, abrange diversos aspectos (subjetivos: sentimentos e emoções manifestadas pelo paciente; e objetivos: dados clínicos do paciente) e respalda ética e legalmente 0 profissional responsável pelo cuidado, uma vez que oferece informações referentes à assistência prestada pela equipe de enfermagem e dados administrativos ${ }^{3}$.

A anotação deve ser valorizada, uma vez que é um dos meios para avaliar o cuidado prestado ao cliente a partir da adoção de indicadores de qualidade que mensuraram tanto 0 processo como os resultados da assistência de enfermagem, existindo correlação positiva entre os registros e a qualidade do cuidado ${ }^{3-5}$.

As informações descritas devem refletir 0 atendimento e o tratamento prestado durante a internação, destacando a evolução da doença, reações e preocupações do paciente, entre outros itens, utilizando-se de termos que expliquem os fatos de maneira clara. Além disso, o registro completo das anotações de enfermagem, com hora e rubrica do profissional responsável pelo mesmo, consiste em responsabilidade ética, determinada no Código de Ética ${ }^{6}$, bem como na Resolução Cofen-358/2009 que dispõe sobre a sistematização da assistência de enfermagem e a implementação do processo da categoria ${ }^{7}$.

A avaliação dos registros permite identificar os pontos fracos que necessitam de melhoria dentro do processo de trabalho em enfermagem, e, ao se executar este processo, toma-se como instrumento de trabalho a auditoria como revisão detalhada das anotações de enfermagem que visa, a partir de diagnósticos realizados, suscitar medidas de aprimoramento do cuidado ${ }^{89}$.

Entretanto, estudos relativos às anotações de enfermagem pontuam as dúvidas e incertezas quanto à qualidade e à validade dos registros e à forma como elas têm sido conduzidas até o momento".

Ressalta-se que a anotação de enfermagem é um instrumento essencial para subsidiar a evolução e a prescrição de enfermagem e, desta forma, garantir a continuidade da assistência e, ainda, legitimar o trabalho do profissional que presta o cuidado ${ }^{1}$.
Estudos demonstram ${ }^{1,4,6}$ que a documentação das intervenções de enfermagem é um dos componentes mais deficientes no processo de assistência de enfermagem, fato que pode estar relacionado ao número reduzido de trabalhadores em relação às necessidades dos pacientes e à falta de tempo para registrar a assistência prestada ${ }^{1}$.

Desta forma, reconhecendo a relevância da veracidade dos registros, este estudo teve por objetivo avaliar a qualidade das anotações de enfermagem realizadas em unidade semiintensiva de um Pronto Atendimento Público e Universitário.

\section{METODOLOGIA}

Trata-se de um estudo descritivo, retrospectivo, de natureza quantitativa, realizado com prontuários de pacientes internados na Unidade de Cuidados Intermediários (UCI) de um Pronto Atendimento (PA) da região Sul do Brasil. Este hospital, credenciado ao Sistema Único de Saúde (SUS), possui 123 leitos e é referência em atendimento de urgência e emergência para região noroeste do Paraná, prestando atendimento nas diversas especialidades clínicas.

A UCl em questão conta com um serviço de atendimento semi-intensivo, destinado a pacientes críticos que aguardam uma vaga para internação em Unidade de Terapia Intensiva (UTI) no mesmo ou em outro hospital conveniado ao Sistema Único de Saúde (SUS), ou ainda a estabilização do quadro clínico visando uma possível transferência para enfermaria. A sistematização da assistência de enfermagem (SAE) é aplicada em todas as fases preconizadas pelo Cofen ${ }^{7}$, apenas neste setor do PA.

Esta unidade possui quatro leitos hospitalares com suporte de UTI, de modo que os pacientes internados apresentam graus diferentes de gravidade e, consequentemente, de cuidado. Nela, os pacientes recebem assistência de enfermagem realizada por apenas um enfermeiro e um técnico em enfermagem por período. 0 número reduzido de pessoas trabalhando na UCl exige uma divisão do trabalho. Assim, os cuidados de enfermagem possíveis de programação são divididos por turno de trabalho. Um exemplo desta divisão é a distribuição do horário de banho dos pacientes por leito, ou seja: nos pacientes do leito $A$ e B, o banho é eito no período da manhã; no período vespertino e noturno, são realizados, respectivamente, os banhos dos pacientes do leito $C$ e $D$. Cabe salientar que nestes dois períodos também é realizada a visita de familiares, o que restringe o horário para a realização de cuidados.

Cabe salientar, ainda, que o enfermeiro da UCI também é responsável pela sala de emergência do PA. Nesta outra unidade, com quatro leitos, a assistência é prestada por apenas um técnico em enfermagem. É importante salientar que, nesta sala, o número de pacientes é variável, além de apresentar diferentes graus de gravidade, pois ela constitui porta aberta para 0 atendimento de urgência e emergência e, sempre que necessário, os pacientes ficam em macas. Estas características fazem com que o enfermeiro permaneça maior parte do seu 
turno de trabalho na sala de emergência, o que, por sua vez, faz com que ele deixe de ser presença constante dentro da UCl.

A realização da SAE é complexa, envolve diversas etapas e instrumental. Para facilitar sua implementação, no local de pesquisa é utilizado um instrumento do tipo quadro pré-estruturado, contendo informações relacionadas aos cuidados que devem ser prestados e registrados nas 24 horas do dia, para os casos de pacientes que necessitam de cuidados semi-intensivos, denominado Plano Assistencial de Enfermagem, uma das etapas da SAE.

De um lado do impresso (folha tamanho $40 \mathrm{~cm}$ ' 30 $\mathrm{cm}$ ) há um quadro para identificação do paciente (nome, idade, sexo, leito, clínica, data da internação, diagnóstico médico e problemas ativos/inativos), um quadro grande dividido em três turnos (manhã, tarde e noite) para o registro de hora em hora ou a cada duas horas de sinais vitais (pressão arterial, pulso, temperatura, respiração, saturação de $0_{2}$, pressão venosa central) e controle hídrico (ganhos = dieta oral e enteral, soro, e medicamentos e perdas = diurese, evacuações, sonda nasogástrica e drenos). Há ainda dois quadros pequenos, um para o registro dos antibióticos em uso (nome, dose, início e término) e outro para o registro da data/hora de instalação/ troca de alguns procedimentos/equipamentos (respirador, tubo orotraqueal, traqueostomia, cateter venoso central, sonda nasogástrica, sonda nasoenteral, sonda vesical de demora, drenos e circuito respiratório.

No verso da folha, são três quadros: um para a prescrição de enfermagem com uma relação de 18 itens de cuidados a serem realizados, os quais são precedidos de parênteses para ser assinalada a sua necessidade e por espaço para o registro do horário em que cada um deles foi realizado; um quadro para o registro da evolução da enfermagem; e outro, dividido em três turnos, para o registro do relatório de enfermagem.

A SAE é uma exigência para as instituiç̧ões de saúde públicas e privadas de todo o Brasil, conforme resolução do Cofen n. 358/20097, sendo também uma orientação da lei do exercício profissional da enfermagem (Lei 7.498, de 25/06/ 86). A implantação da SAE torna-se uma estratégia para a organização da assistência de enfermagem nas instituições ${ }^{8-9}$.

Os dados foram coletados em março de $2010 \mathrm{em}$ prontuários de 16 pacientes internados na unidade nos meses de dezembro de 2009 e janeiro de 2010. 0 único critério utilizado para inclusão no estudo é que o paciente estivesse cadastrado na Central de Leitos para uma vaga na UTI. Foram auditados um total de 90 Planos Assistenciais de Enfermagem.

Para a coleta de dados foi utilizada a parte três do Formulário para Avaliação da Qualidade do Cuidado de Enfermagem Prestado em UTI ${ }^{4}$, a qual consta do Roteiro de Auditoria Retrospectiva (preenchido por meio da Busca Ativa nas Anotações de Enfermagem) composto por seis itens: I. Dados de Identificação (com três subitens), Il. Prescrição de Enfermagem (com nove subitens), III. Procedimentos de Enfermagem (com treze subitens), IV. Registros de Enfermagem (também com treze subitens), V.
Execução de Ordens Médicas (com quatro subitens); VI . Unidade de Terapia Intensiva Adulto (com oito subitens). Cada subitem do Roteiro de Auditoria Retrospectiva tem cinco opções de resposta: não se aplica; preenchimento completo; incompleto, incorreto e não preenchido. Para o presente estudo, as respostas foram agrupadas em três opções: completo/correto, incompleto/incorreto enão preenchido.

Para a classificação da qualidade do preenchimento das anotaç̃̃es de enfermagem, consideraram-se os mesmos critérios/padrões e percentuais propostos pela autora ${ }^{3}$ do instrumento, a qual considera boa qualidade quando mais de $80 \%$ dos aspectos integrantes de um item são preenchidos completa e/ou corretamente; entre $5 \%$ a $15 \%$ de forma incompleta e/ou errada e menos de $5 \%$ não são preenchidos.

Para a identificação dos percentuais referentes ao preenchimento, os dados de cada um dos dias de internação foram registrados em uma planilha no Excel, construída especificamente para este fim; em seguida, com base no número de dias/vezes que a atividade foi registrada e na forma como 0 registro foi feito (completo/correto, incompleto/incorreto e não preenchido), calculou-se o percentual alcançado em cada uma das modalidades de preenchimento. Em seguida, somaram-se os percentuais alcançados em cada um dos subitens e, então, aplicou-se a regra de três para obter o percentual alcançado em cada um dos seis itens do instrumentos.

Os dados foram compilados e tratados em banco de dados no programa computacional Excel Access 2003, e apresentados em forma de tabela com suas respectivas porcentagens de preenchimento.

0 desenvolvimento do estudo ocorreu em conformidade com o preconizado pela resolução 196/96 do CNS - MS, ${ }^{10}$ que disciplina pesquisa com seres humanos, e seu projeto foi aprovado pelo Comitê Permanente de Ética em Pesquisa com Seres Humanos da Universidade Estadual de Maringá-PR (Parecer $n^{0}$ 624/2009), que dispensou o uso do Termo de Consentimento Livre e Esclarecido, uma vez que os dados seriam obtidos em fontes secundárias.

\section{RESULTADOS E DISCUSSÃO}

Por compreender que o conhecimento das características da população às quais se destinam os cuidados de enfermagem é importante para o processo de avaliação da qualidade, a seguir serão descritos os dados de caracterização clínica e demográfica dos pacientes.

Participaram deste estudo 16 pacientes, cuja amplitude de idade variou de 28 a 86 anos, com média de 58 anos e mediana de 62 anos, sendo a maioria do sexo feminino (10\% a $62,5 \%$ ). No que se refere ao diagnóstico de internação, seis pacientes $(37,5 \%)$ apresentaram problema neurológico (acidente vascular encefálico, traumatismo craniano e crise convulsiva); seis (37,5\%), problemas relacionados ao sistema respiratório (edema agudo de pulmão e insuficiência 
respiratória), e quatro (25\%); problemas cardíacos (insuficiência cardíaca congestiva e choque cardiogênico). 0 tempo médio de permanência na unidade semi-intensiva foi de oito dias, cuja amplitude variou de 1 a 20 dias.

0 Percentual de Preenchimento (PP) Completo, Incompleto e Não Preenchido, obtido para cada um dos itens do Roteiro de Auditoria Retrospectiva que avaliaram os registros de enfermagem realizados no prontuário do paciente em recuperação na UCl, está disposto na Tabela 1. Observa-se que, dos aspectos avaliados no período auditado, nenhum atingiu um bom padrão de qualidade, e que o maior problema está em não preencher o item, com exceção do item VI - Unidade de Terapia Intensiva, em que a proporção de aspectos incompletos foi bem maior do que não preenchidos.

Tabela 1 - Percentual de preenchimento dos registros de enfermagem realizados em unidade semi-intensiva de um hospital universitário do nor te do Paraná, 2010.

\begin{tabular}{|c|c|c|c|}
\hline \multirow[t]{3}{*}{ Itens d a audiforia retrospectiva } & \multicolumn{3}{|c|}{ Preenc himento } \\
\hline & Completo & Inc ompleto & Nã̃ preenchido \\
\hline & $\%$ & $\%$ & 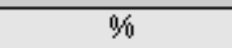 \\
\hline I. Dados de identificação & 74,8 & 18,9 & 6,3 \\
\hline II. Prescrição de enfermagem & 39,3 & 16,3 & 44,3 \\
\hline III. Procedime ntos de enfermagem & 41,1 & 21,1 & 37,6 \\
\hline IV. Reg is tros de enfermagem & 54,7 & 179 & 27,5 \\
\hline V.Execução de orders médicas & 25,0 & 21,4 & 53,6 \\
\hline VI. Unidade de Terapia Intensiva & 34,9 & 562 & 9,0 \\
\hline Média & 45,0 & 25,3 & 29,7 \\
\hline
\end{tabular}

No Item I - Dados de Identificação, nota-se que, apesar dos PP não atingirem os critérios de qualidade propostos, não se distanciaram muito dos valores considerados satisfatórios.

Fizeram parte deste item três subitens: preenchimento correto dos dados de identificação do paciente; preenchimento completo dos dados de identificação do paciente; registro de data, hora, n. $^{\circ}$ COREN (Conselho Regional de Enfermagem) e assinatura do enfermeiro na prescrição de enfermagem.

Dos subitens referidos, destacou-se a presença de data, hora, n. ${ }^{\circ}$ COREN e assinatura do enfermeiro na prescrição de enfermagem que apresentou um PP Incompleto de $37 \%$ e Não Preenchido de $18,9 \%$.

A ausência de registro do horário impossibilita saber se a prescrição foi realizada no início ou no final do plantão e também se contemplou as alterações que o paciente apresentou no decorrer deste período. 0 registro do nome e o carimbo do enfermeiro e do técnico de enfermagem que prestaram atendimento possibilitam a identificação dos profissionais, na vigência de possíveis dúvidas e questionamentos subsequentes ao atendimento ${ }^{11}$.

A qualidade dos registros sobre 0 atendimento prestado aos pacientes em situações de emergência é uma preocupação crescente nas instituições, considerando que informações insuficientes afetam e interpõem-se à qualidade do cuidado realizado, principalmente quando relacionado aos dados de identificação que, quando incompletos, podem comprometer a segurança do paciente.

0 registro de todas as etapas da SAE deve ser avaliado em virtude da sua importância como facilitador da valorização da assistência de enfermagem a ser estabelecida para o paciente ${ }^{12,13}$. Na tentativa de obtenção de um cuidado adequado ao paciente crítico, é preciso haver uma estrutura organizacional especíica quanto aos cuidados humanos e aos recursos físicos e materiais. É pela situação instável deste tipo de cliente que a assistência de enfermagem é ainda mais necessária, visto que facilitará o domínio apurado da técnica, conciliando-a com o cuidado humanizado e holístico. Quanto maior o número de necessidades afetadas pelo cliente, maior é a necessidade de se planejar a assistência visando à organização, à eficiência e à validade da assistência prestada ${ }^{14}$.

Há dificuldades especiais para a assistência de enfermagem nos hospitais, que advêm da necessidade de conciliação entre as atividades assistenciais e gerenciais, devido ao aumento do fluxo e gravidade dos pacientes e da dificuldade de manter a quantidade necessária da equipe de enfermagem ${ }^{12}$.

0 item Prescrição de Enfermagem obteve 39,3\% de PP Completo, porém o valor encontrado ficou muito abaixo do esperado (PPe"80\%), não sendo considerado de boa qualidade.

Este item foi composto por nove subitens relacionados com a prescrição de enfermagem, em que foram observados dois subitens com elevado PP "Incompleto": a) a primeira prescrição é feita pela mesma enfermeira que o admitiu (PP $50 \%$ ) eb) os cuidados prescritos foram checados e rubricados (PP 61,1\%).

Com relação à presença de rubrica e checagem dos cuidados prescritos pelo enfermeiro, o elevado PP Incompleto pode decorrer do número insuficiente de recursos humanos, e 


\section{Unidades semi-intensivas: qualidade das anotações de enfermagem}

também do fato de que, normalmente, são prescritos apenas cuidados de rotina, os quais a equipe desempenha independente de terem sido prescritos ou não.

Apesar de ser uma atividade privativa do enfermeiro ${ }^{15}$, a prescrição de enfermagem, paraser realizada integralmente, necessita da colaboração de toda a equipe de enfermagem, uma vez que o planejamento da assistência é desenvolvido pelo enfermeiro, mas o cuidado ao paciente tem sido executado principalmente pelo pessoal de enfermagem de nível médio. A falta de registro constitui um obstáculo para sua execução, sendo fundamental que não só o enfermeiro passe por orientação e capacitação, mas toda a equipe, possibilitando assim que compreendam sua importância e colaborem para a sua execução de forma efetiva.

Ainda para o item Prescrição de Enfermagem, o PP Não Preenchido foi 44,3\%, não correspondendo ao estabelecido (menor que $5 \%$ ), o que representou em todos os subitens avaliados percentuais muito elevados de Não Preenchimento. Isso demonstra que não está havendo uma boa qualidade nos registros no que diz respeito à prescrição de enfermagem, o que pode estar afetando 0 cuidado, ou seja, a realização da assistência individualizada, baseada nas necessidades humanas básicas afetadas de cada paciente.

Vale ressaltar que a prescrição de enfermagem é o conjunto de medidas decididas pelo enfermeiro, que direciona e coordena a assistência ao paciente de forma individualizada e contínua, objetivando a prevenção, promoção, proteção, recuperação e manutenção da saúde ${ }^{13-14,16}$.

Considerando a importância da SAE no cotidiano da enfermagem, observa-se a necessidade de capacitar melhor profissionais para a execução, trabalhando com instrumentos aplicáveis a cada realidade para oferecer um cuidado integral e qualificado ao paciente.

Quanto ao item denúmero III-Procedimentos de Enfermagem, os PPCompleto, Incompleto e Não Preenchido também se mantiveram fora dos valores esperados. Destaca-se elevado índice nos subitens: a) anotação e controle das evacuações, com 62,1\% de PP Incompleto e b) registro de episódios eméticos, com 98,3\% de PP Não Preenchido. Lembrando-se que a anotação tanto da ingestão como da eliminação de líquidos é importante para o controle do balanço hídrico do paciente.

0 cálculo diário, ou mesmo a cada plantão, do balanço hídrico do paciente, subsidia a tomada de decisão médica e a redefinição de terapêuticas e de cuidados. A importância desta prática na terapia intensiva acentua-se visto que tênues variações de condutas, fundamentadas em dados incompletos do balanço hídrico, podem gerar consequências indesejáveis devido à gravidade do paciente ${ }^{17}$.

0 registro inadequado da realização dos procedimentos de enfermagem pode colocar em dúvida a sua realização, visto que, se não existe registro, não há como se garantir que foi executado. Além de motivar questionamentos sobre a realização do procedimento, o registro não realizado de forma adequada promove falha na comunicação entre as equipes de enfermagem, comprometendo, assim, a continuidade do cuidado ${ }^{17}$.
0 item IV - Registros de Enfermagem obteve $54,7 \%$ de PP Completo. Apesar de ter sido o segundo item que mais se aproximou do valor estabelecido (PPe"80\%), não é possível considerá-lo como sendo de qualidade, uma vez que o percentual obtido está muito abaixo do preconizado para este estudo.

Dentre os subitens referidos, os que apresentaram elevado PP Incompleto em relação ao preconizado para este estudo foram: a) as anotações indicam quem realizou o cuidado $(P P=64,4 \%)$ e b) há horário e rubrica em cada anotação ( $\mathrm{PP}=38,9 \%)$, concordando com os resultados encontrados no item Prescrição de enfermagem.

Diante da necessidade da existência de pelo menos uma anotação a cada período de seis horas, como é padronizado na instituição, esta ação é realizada enquanto cumprimento de tarefa, sem o compromisso com a qualidade dos registros.

Com relação ao PP Não Preenchido, também se mostrou elevado nos subitens: a) prescrição indica ações relacionadas ao atendimento de necessidades psicobiológicas eb) aspecto e evolução de lesões cutâneas ( $\mathrm{PP}=68,2 \%$ ).

A realização de registros incompletos sobre os cuidados com lesões cutâneas, além de caracterizar falta de compromisso e omissão de cuidado por parte da equipe de enfermagem, interfere no tratamento das lesões, visto que para a sua continuidade são necessários registros completos sobre as condutas tomadas e a evolução diária das suas características ${ }^{18}$. Estas informações possibilitam a decisão de manter o cuidado ou a necessidade de substituir.

No item V - Execução das ordens médicas, as anotações não foram consideradas de qualidade, pois apresentaram o menor PPCompleto, que foi de 25\%. 0 PP Incompleto também não atingiu o objetivo com $21,4 \%$, sendo que o estabelecido é menor que $15 \%$.

0 PP Não Preenchido foi de 53,6\% com ênfase nos subitens: a) as solicitações médicas foram transcritas para a prescrição de enfermagem e b) a prescrição médica indica conhecimento das ações de enfermagem.

0 item VI - Unidade de Terapia Intensiva apresentou $34,9 \%$ de PP Completo. Os registros referentes a este item também não foram considerados de qualidade pelo fato de que o PP obtido não atingiu o valor considerado de qualidade (acima de $80 \%$ ). Ao contrário o PP Incompleto, pela primeira vez, ficou dentro dos esperados $9 \%$, sendo que o estabelecido é menos que $15 \%$.

0 PP Não Preenchido foi de 56,2\% configurando-se como elevado, com destaque aos subitens: a) registros de pressão arterial média (PAM) ( $\mathrm{PP}=100 \%$ ) pelo menos uma vez por período, b) registros de pressão venosa central pelo menos uma vez por período (100\%) ec) registros de mudança de decúbito programada ( $\mathrm{PP}=67,9 \%)$.

Infere-se que o não preenchimento do valor da PAM pode ser decorrente da ausência do equipamento apropriado, pelo desconhecimento da forma de calcular a PAM, ou ainda, pela pouca valorização deste dado para a avaliação do paciente. 
A necessidade de se verificar a PAM justifica-se porque este dado consiste em um componente básico da monitorização hemodinâmica, quando há necessidade de vigilância contínua das variações da pressão arterial. Isso ocorre principalmente quando o paciente está em uso de medicamentos vasoativos ou necessitando de reposição da volemia, visto que a PAM possibilita um acompanhamento mais fidedigno das condições de gravidade dos pacientes ${ }^{16}$.

Com relação à ausência de preenchimento referente à mudança de decúbito, acredita-se que seja devida ao fato de este cuidado ser checado na prescrição de enfermagem. Sendo assim, os integrantes da equipe podem considerar que é desnecessário repetir esta informação no relatório.

Estes resultados são semelhantes aos encontrados em pesquisa realizada na mesma instituição ${ }^{1}$ por meio da análise de cinco prontuários de pacientes que encontra-se em acompanhamento terapêutico intensivo e posteriormente foram encaminhados para as unidades de internação, o qual mostrou que a maioria dos registros encontrava-se incompleto, não permitindo o fornecimento de dados que subsidiem a continuidade do processo de cuidado ao paciente.

Um pesquisa realizada em unidades de internação e Unidade de Terapia Intensiva de um hospital escola no interior do Paraná, em 2004, revelou que a clínica cirúrgica foi a que atingiu o número de anotações de enfermagem desejável, 0 que denota a falta de comprometimento com os registros de enfermagem realizados em UTI, que deveriam ser criteriosamente executados e com maior frequência relatando todos os acontecimentos com os pacientes gravemente enfermos ${ }^{19}$.

A comunicação escrita na forma de anotações de enfermagem é essencial, uma vez que a prática do cuidado gera inúmeras informações que não devem ser perdidas, mas sim registradas. Sendo assim, a contínua capacitação e 0 aprimoramento do processo de registros das condutas de enfermagem são necessários para a garantia da qualidade do cuidado ${ }^{20}$.

\section{CONSIDERAÇÕES FINAIS}

Os resultados do estudo chamam a atenção do enfermeiro para problemas que se manifestam, praticamente, em todas as unidades de assistência ao paciente, pois mostram que nenhum dos seis itens sob avaliação alcançou o critério de bom padrão de qualidade em seu preenchimento; 0 item I - Identificação do paciente foi o que esteve mais próximo de alcançar esta qualidade e o item V - Execução de ordens médicas, o que esteve mais distante. Mostrou-se, ainda, que o maior problema está na ausência de informações, com exceção do item VI - Unidade de Terapia intensiva em que a proporção de aspectos incompletos foi bem maior do que não preenchidos.

Considerando que a realização de anotações completas, claras e precisas referentes aos procedimentos realizados com o paciente contribui para melhora na qualidade da assistência, favorecendo a continuidade do cuidado e a legitimação do trabalho de enfermagem, e ainda que todas as fases da SAE precisam ser realizadas, uma vez que elas são interdependentes, os resultados do estudo mostram a necessidade de um acompanhamento mais amiúde dos registros efetuados no dia a dia e também da realização de cursos de educação em serviço para todos os membros da equipe de enfermagem atuantes neste setor, de modo a sensibilizá-los quanto à importância de registrarem informações relacionadas à condição do paciente e, em especial, às atividades e ações realizadas para seu atendimento.

É importante destacar a importância de uma melhor documentação das ações e intervenções realizadas pela equipe de enfermagem, lembrando que os registros devem traduzir o máximo de conhecimento sobre as condições de saúde dos indivíduos, incluindo-se tanto os aspectos referentes a procedimentos quanto as necessidades, queixas e evolução dos pacientes, pois as anotações comprovam o trabalho da enfermagem, sendo função dos enfermeiros liderar e acompanhar a efetivação desse registro.

No campo da pesquisa e do desenvolvimento do conhecimento, os resultados encontrados apontam também para a necessidade de realização de mais estudos, de modo que se possa compreender melhor os fatores relacionados com a deficiência nos registros de enfermagem. No caso específico deste serviço, seria importante investigar com os profissionais da equipe de enfermagem, os motivos relacionados com registro incompleto ou ausentes, com vistas a instituir medidas que contemplem as dificuldades apontadas e, assim, obter qualidade nos registros efetuados.

Consideramos que o pouco tempo de auditoria realizada nos prontuários pode constituir-se em uma limitação importante do estudo. Cabe salientar que, embora no período auditado (dezembro e janeiro), o setor não contasse com a presença de alunos de graduação em enfermagem, o que teoricamente justificaria a ausência e incompletude dos instrumento, ainda assim ele pode ser considerado atípico, pois é um período do ano em que são mais frequentes as férias e ausências de funcionários por motivos diversos, havendo necessidade de substituições. Contudo, o levantamento efetuado não permite perceber se os resultados encontrados foram ou não influenciados por características do paciente que esteve internado no período, pela falta de capacitação técnica dos profissionais ou mesmo pelo dimensionamento de pessoal reduzido, bem como pela estrutura organizacional do serviço onde o estudo foi realizado.

Por fim, salientamos que seria amadorismo acreditar que a implantação e a manutenção da SAE são fáceis; pelo contrário, as dificuldades são grandes e só serão superadas a partir de um esforço conjunto de todos os membros da equipe de enfermagem atuantes no setor. 


\section{REFERÊNCIAS}

1.Matsuda LM, Silva DMP, Évora YDM, Coimbra JAH. Anotações/registros de enfermagem: instrumento de comunicação para a qualidade do cuidado. Rev Eletr Enferm. [on-line] 2006 set; [citado 2010 nov 18]; 8(3): 415-21. Disponível em: http://www.fen.ufg.br/revista/revista8 3/v8n3a12.htm

2.Venturine DA, Marcon SS. Anotações de enfermagem em uma unidade cirúrgica de um hospital escola. Rev Bras Enferm. 2008; 61(5): 570-77.

3. D'Innocenzo M, Feldman LB, Fazenda NRR, Helito RAB, Ruthes RM. Indicadores, auditorias, certificações- ferramentas de qualidade para gestão em saúde. São Paulo: Martinar; 2006.

4. Haddad MCL. Qualidade da assistência de enfermagem: o processo de avaliação em hospital universitário público [tese]. Ribeirão Preto: Escola de Enfermagem, Universidade de São Paulo; 2004.

5. Setz VG, D'Innocenzo M. Avaliação da qualidade dos registros de enfermagem no prontuário por meio da auditoria. Acta Paul Enferm. 2009 maio/jun; 22(3): 313-17.

6. D'Innocenzo M, Adami NP. Análise da qualidade dos registros de enfermagem nos prontuários de pacientes de hospitais de ensino e universitários. Acta Paul Enferm. 2004 out/dez; 17(4): 383-91.

7. Conselho Federal de Enfermagem. Resolução n 358 , de 15 de outubro de 2009. Dispõe sobre a Sistematização da Assistência de Enfermageme a implementação do processo de enfermagem em ambientes, públicos ou privados, em que ocorre o cuidado profissional de Enfermagem, e dá outras providências. Disponível em: http://site.portalcofen.gov.br/node/ 4384

8. Luz A, Martins AP, Dinewicz AM. Características de anotações de enfermagem

encontradas em auditoria. Rev Eletr Enferm. [on-line] 2007 maio; [citado 2010 out 16]; 9(2): 334-61. Disponível em: http://www.fen.ufg.br/revista/ v9/n2/v9n2a05.htm

9. Gonçalves LRR, Nery IS, Nogueira LT, Bonfim EG. 0 desafio de implantar a sistematização da assistência de enfermagem sob a ótica de discentes. Esc Anna Nery. 2007 set; 11(3): 459-65.

10. Ministério da Saúde (BR). Conselho Nacional de Saúde. Resolução n. 196, de 10 de outubro de 1996. Dispõe sobre as diretrizes e normas regulamentadoras de pesquisas envolvendo seres humanos. Brasilia(DF); 1996. Disponível em: http://dtr2004.saude.gov.br/susdeaz/legislacao/ arquivo/Resolucao_196_de_10_10_1996.pdf

11. Saleh CMR. Sistematização da assistência de enfermagem no prontosocorro. In: Calil AM, Patanhos WY. 0 enfermeiro e as situações de emergências. São Paulo: Atheneu; 2007. p.45-55.

12. Repetto MA, Souza MF. Avaliação da realização e do registro da Sistematização da Assistência de Enfermagem-SAE em um hospital universitário. Rev Bras Enferm. 2005 maio/jun; 58(3): 325-29.

13. Padilha EF. Auditoria como ferramenta para a qualidade do cuidado de enfermagem em unidade de terapia intensiva de um hospital universitário [dissertação] Maringá: Universidade Estadual de Maringá; 2010.
14. Bittar DB, Pereira LV, Lemos RCA. Sistematização da assistência de enfermagem ao paciente crítico: proposta de instrumento de coleta de dados. Texto\&Contexto Enferm. 2006 out/dez; 15(4): 617-28.

15. Conselho Federal de Enfermagem. Resolução n ${ }^{0} 272$, de 27 de agosto de 2002. Dispõe sobre a Sistematização da Assistência de Enfermagem-SAE- nas Instituições de Saúde Brasileiras. Disponível em: http://site.portalcofen.gov.br/node/4309

16. Horta VA. Processo de enfermagem. São Paulo: Universidade de São Paulo; 1979.

17. Andrade CR, Chianca TCM, Werli AR, Couto CR. Avaliação da qualidade do registro do balanço hidroeletrolítico. Rev Enferm Hosp. [on-line] 2009 jul./dez; [citado 2010 nov 20]; 1(1): 3-4. Disponível em: http://www.enf.ufmg.br/ojs/index.php/reonline/article/viewFile/8/17

18. Vituri D, Matsuda L. Analysis of nursing records as to care quality: a retrospective descriptive exploratory study of quantitative approach. Online Brazilian Journal of Nursing [on-line]. 2008 Feb; [citado 2010 nov 15]; 7(1). Disponível em: http://www.objnursing.uff.br/index.php/ nursing/article/view/1241

19. Matsuda LM, Carvalho ARS, Évora YDM. Anotações / registros de enfermagem em um hospital-escola. Cienc Cuid Saude. 2007; 6 (2 supl): $337-46$.

20. Corrijo AR, Oguisso T. Trajetória das anotações de enfermagem: um levantamento em periódicos nacionais 1957-2005. Rev Bras Enferm. 2006; 56( n esp): 454-58. 